Etnográfica

Revista do Centro em Rede de Investigação em

Antropologia

vol. $23(3) \mid 2019$

Vol. 23 (3)

\title{
Aproximación etnográfica a las manifestaciones de agencia en personas nonagenarias y centenarias en Chile
}

An ethnographic approach to agency manifestations in nonagenarians and centenarians in Chile

Paulina Osorio-Parraguez, Ignacia Navarrete y Samuel Briones

\section{(2) OpenEdition}

\section{Journals}

Edición electrónica

URL: https://journals.openedition.org/etnografica/7400

DOI: 10.4000/etnografica.7400

ISSN: 2182-2891

\section{Editor}

Centro em Rede de Investigação em Antropologia

Edición impresa

Fecha de publicación: 1 octubre 2019

Paginación: 673-692

ISSN: 0873-6561

Referencia electrónica

Paulina Osorio-Parraguez, Ignacia Navarrete y Samuel Briones, «Aproximación etnográfica a las manifestaciones de agencia en personas nonagenarias y centenarias en Chile», Etnográfica [En línea], vol. 23 (3) | 2019, Publicado el 28 noviembre 2019, consultado el 20 enero 2022. URL: http:// journals.openedition.org/etnografica/7400 ; DOI: https://doi.org/10.4000/etnografica.7400

\section{(c) (7) \&}

Etnográfica is licensed under a Creative Commons Attribution-NonCommercial 4.0 International License. 


\section{Aproximación etnográfica a las manifestaciones de agencia en personas nonagenarias y centenarias en Chile}

\section{Paulina Osorio-Parraguez, Ignacia Navarrete}

\section{y Samuel Briones}

Este artículo presenta los resultados de una investigación etnográfica cuyo objetivo es conocer las manifestaciones de agencia de personas nonagenarias y centenarias en su proceso y experiencia de envejecimiento avanzado. Se abordan las particularidades de la agencia en la longevidad en determinados contextos, que configuran la experiencia de envejecer de los sujetos. La hipótesis de trabajo ha sido que, al profundizar en el conocimiento cualitativo del conjunto de variaciones que tiene la vida de las personas de la cuarta edad, emergen una serie de manifestaciones de agencia, y esta capacidad no se reduce/pierde, sino más bien se manifiesta de forma diversa y particular a la expresada en otros momentos del curso de vida. La investigación es de carácter descriptivo-explicativo y se enmarca dentro de una aproximación cualitativa-inductiva de producción y análisis de la información. La estrategia metodológica fue la etnografía, con énfasis en observación directa y realización de entrevistas en profundidad. Se realizaron visitas etnográficas a personas nonagenarias y centenarias en zonas rurales y urbanas de Chile. Las manifestaciones de agencia identificadas en este artículo generan acciones bajo cuatro formas diferentes: movilizar; hacer/no hacer; resistir posición; y normalizar posición. Los resultados muestran que no necesariamente hay una relación entre el deterioro de las capacidades físicas y funcionales y la reducción/pérdida de agencia, sino que se trata del contexto de las relaciones sociales y las diversas posiciones estructurales que adquieren los más envejecidos lo que inhibe o constriñe las manifestaciones de agencia en la cuarta edad.

PALABRAS-CLAVES: etnografía, nonagenarios, centenarios, agencia, cuarta edad, longevidad.

An ethnographic approach to agency manifestations in nonagenarians and centenarians in Chile - This paper presents the results of an ethnographic research aimed to study agency manifestations of nonagenarians and centenarians in their process and experience of advanced aging. Agency particularities of longevity are addressed in contexts that shape the aging experience of subjects. The working hypothesis has been that by producing deep qualitative data about the lives of people living in the fourth age, a series of agency manifestations emerge that show that this capacity is not reduced/lost, but rather is transformed in various ways that are specific to this moment of the life course. The research is of descriptive-explanatory nature and is framed within a qualitative-inductive approach of 


\begin{abstract}
data production and analysis. The methodological strategy put emphasis on direct observation and conduction of in-depth interviews. Ethnographic visits were made to nonagenarians and centenarians in rural and urban areas of Chile. The agency manifestations identified in this article generate actions in four different domains: mobilization; to do/not-to do; resist position; and normalize position. The results show that there is not necessarily a relationship between the deterioration of physical and functional capacities and the reduction/loss of agency. The context of social relations and the various structural positions acquired by older people inhibits or constrains the manifestations of agency in the fourth age
\end{abstract}

KEYWORDS: ethnography, nonagenarians, centenarians, agency, fourth age, longevity.

OSORIO-PARRAGUEZ, Paulina (posorio@uchile.cl) - Departamento de Antropología, Universidad de Chile, Chile.

NAVARRETE, Ignacia (ignav.lu@gmail.com) - Universidad de Chile, Chile.

BRIONES, Samuel (sbrionesb@gmail.com) - Universidad de Chile, Chile.

\title{
INTRODUCCIÓN
}

Una característica importante de las sociedades en la actualidad es su acelerado envejecimiento poblacional. ${ }^{1}$ La población de América Latina y el Caribe está envejeciendo paulatina pero inexorablemente; se espera que la población de 60 años o más se triplique entre 2000 y 2050. El envejecimiento demográfico implica el aumento de la proporción de personas mayores en las poblaciones y el aumento de la expectativa de vida. Así, la longevidad se presenta como una característica del envejecimiento general de la población, vale decir, que no sólo hay más población mayor, sino que ésta vivirá más años de vejez, aumentando significativamente las personas de la cuarta edad. En esta transición demográfica, Chile se presenta como uno de los cuatro países más envejecidos de la región y con una creciente longevidad de su población mayor. Según datos censales de 2017, en Chile viven cerca de 80.000 personas mayores de 90 años (INE 2017) y para el 2050 este grupo de edad aumentará a cerca de 360.000 personas, de las cuales el 73\% serán mujeres (USCB 2018).

I Trabajo realizado en el marco del proyecto Fondecyt 1150823, "Envejecimiento y longevidad: estudio etnográfico sobre personas nonagenarias y centenarias en Chile", al cual están vinculados Ignacia Navarrete y Samuel Briones. Agradecemos el trabajo de Javiera Chahuán Pérez y José Pinto Galaz, cuyos relatos etnográficos se encuentran en este artículo; a todo el equipo del proyecto Fondecyt 1150823, por sus comentarios y observaciones críticas, las cuales contribuyeron al desarrollo de este trabajo; y a las personas nonagenarias y centenarias, y a sus familias, que participaron en el proyecto y posibilitaron esta investigación. 
Los mayores de 90 años es el grupo de edad de más rápido crecimiento a nivel mundial y se espera que a mediados de siglo, al menos la mitad de estas personas sufrirá algún tipo de demencia o vivirá en situación de discapacidad (Kawas 2008). Extender el período de la vida en que las personas mayores de 90 años gozan de buena salud y están libres de discapacidad es uno de los desafíos más importantes de las ciencias biomédicas y gerontológicas.

La comprensión de esta etapa de la vida, a partir de abordajes cualitativos, que permitan profundizar en un conocimiento socio-antropológico de la longevidad, es fundamental. La cuarta edad se la ha definido a partir de los 80 años de edad (Lalive d'Epinay y Spini 2007) y se ha configurado como una etapa vital cruzada por imaginarios de fragilidad, dependencia y enfermedad (Gilleard y Higgs 2011). Esto significa un desplazamiento del sujeto en la estructura social y la pérdida de agencia sobre el cuerpo, la propia vida y los entornos sociales cercanos. En este sentido, socialmente se daría una igualación de la noción de agencia con la de envejecimiento exitoso, al ser una realidad producida individualmente, de manera activa y en buena salud. Por lo tanto, en la cuarta edad se daría una controversia, en cuanto se busca sumar calidad de vida y empoderar a las personas mayores, pero ello no necesariamente se traduciría en una mayor capacidad de agencia en los sujetos longevos. En este marco, nuestra hipótesis de trabajo ha sido que, al profundizar en el conocimiento cualitativo de la diversidad y el conjunto de variaciones que tiene la vida de las personas de la cuarta edad, emerge una serie de manifestaciones de agencia, y ésta no se reduce/pierde, sino más bien se expresa de forma diversa y particular. En virtud de dicha controversia es que el presente artículo busca profundizar en el conocimiento cualitativo de las formas de agencia en personas nonagenarias y centenarias.

\section{LONGEVIDAD: CUARTA EDAD Y CAPACIDAD DE AGENCIA}

Las manifestaciones de agencia que esta investigación indaga se enmarcan en lo que se ha denominado "vejez avanzada" o "cuarta edad", siendo este último término de uso más común en Europa. La cuarta edad nace como un periodo de la vida emergente y discreto en lo que ha sido la vejez de los individuos en las sociedades modernas. El filósofo británico Peter Laslett acuñó el término "cuarta edad" a finales de los años 80, para hacer la distinción entre grupos de viejos sanos y grupos de viejos más propensos a sufrir algún tipo de discapacidad o condición crónica inhabilitante (Laslett 1989). En esta definición, la tercera edad es vista en sus aspectos productivos, saludables y activos de la vejez con la intención de hacer frente a la discriminación basada en la edad. Los aspectos estigmatizantes del envejecimiento sólo fueron desplazados a una edad posterior, la cuarta edad. Así, la cuarta edad comienza a ser un campo de interés para la investigación social (Lalive d'Epinay y Cavalli 2013) 
y considerada como un espacio social donde el poder, el estatus y la ciudadanía ya no pueden ser ejercidos por aquellos que la personifican (Gilleard y Higgs 2010: 121).

Esta diferencia entre ambas etapas de la vida se nutre de la ecuación presente en los imaginarios de las personas acerca de la vejez, que nos dicen que la salud, la actividad y la independencia son necesarias para la agencia, y estas son condiciones que se pueden mantener durante la "tercera edad". Investigaciones sobre la agencia en la tercera edad (Soares 2014) destacan la capacidad de reflexividad de mujeres mayores al dialogar y generar elecciones en sus vidas cotidianas con la estructura normativa de esta edad - como son las prácticas institucionales asociadas al "envejecimiento exitoso" -. Sin embargo, la revisión de bibliografía especializada señala que, con la llegada de la fragilidad y la discapacidad, la agencia se reduciría/perdería, entrando en el dominio de la "cuarta edad". Gilleard y Higgs (2010) utilizan la metáfora del "hoyo negro" (black hole) para retratar la forma en que se estaría conceptualizando la cuarta edad en el curso de vida normativo: un límite que despoja al sujeto de cualquier posibilidad de agencia y que lo relega a un espacio sólo definido por la incapacidad y la cercanía con la muerte. Grenier y Phillipson (2013) señalan que el problema surge al cuestionar la manera en que tradicionalmente se define la agencia en la vejez, relacionada con las ideas de salud y participación que nutren la perspectiva del "envejecimiento exitoso". Estos autores proponen que la discusión en torno a la agencia en la cuarta edad requiere que investigadores cambien su interpretación binaria de la agencia, de "ausente/ presente" hacia la comprensión de formas posibles de agencia, las condiciones estructurales que la inhiben o posibilitan y sus diversas expresiones.

La definición de agencia utilizada en la presente investigación es cercana a la conceptualización de Archer (2003), que considera la agencia como producto de la reflexividad humana y que permite a los sujetos el desarrollo de proyecciones, en relación a medios y fines, y cambios respecto de su posición subjetiva en una estructura objetiva. Asimismo, se consideran los postulados de Navarro (1996), en cuanto entiende que la agencia emerge a partir de una asimetría que rompe la estabilidad del sujeto con la estructura. De acuerdo al autor, la expresión de estas asimetrías estaría orientada a producir una nueva estabilidad entre sujeto y estructura, siendo una característica intrínseca de la agencia. Sin embargo, la agencia de los individuos se encuentra constreñida o propiciada por factores estructurales, los cuales tienen poder de causalidad sobre las manifestaciones de agencia de los individuos (Fletcher 2017). Es en las manifestaciones de agencia de los individuos dentro de contextos estructurales donde este poder de causalidad puede verse actualizado en la realidad o no, dando paso al proceso morfogenético (Archer 1996). Así, reflexividad y decisión para la formulación de proyectos son las dos condiciones basales de la agencia humana según esta conceptualización. 


\section{ESTRATEGIA DE CAMPO PARA LA INVESTIGACIÓN DE LA AGENCIA} EN LA LONGEVIDAD

Al buscar conocer las manifestaciones de agencia en personas nonagenarias y centenarias, en zonas urbanas y rurales de Chile, se optó por un abordaje cualitativo. El trabajo de campo se desarrolló desde septiembre de 2015 hasta noviembre de 2017. El método fue etnográfico, permitiendo conocer potencialidades, estrategias y fortalezas presentes en la experiencia vital de personas longevas. En este marco, la etnografía constituyó una herramienta transversal para la comprensión e interacción con la realidad en estudio, como son las experiencias y relaciones cotidianas de personas nonagenarias y centenarias en sus entornos familiares, sociales e institucionales. También posibilitó acceder a lo sutil de la vida cotidiana de hombres y mujeres mayores, a sus ritmos de vida, observar sus interacciones y relaciones con otros/as, en determinados territorios y ciudades, lo que implicó un ejercicio hermenéutico de interpretación constante y de descripción densa (Geertz 2005).

El paisaje es un elemento importante en la vida cotidiana de cualquier grupo humano; por lo tanto, al aproximarse al contexto geográfico-cultural donde han envejecido y viven su longevidad las personas nonagenarias y centenarias, se evidencian las transformaciones y particularidades de nuestro escenario de investigación. Se trabajó con la observación directa y con las entrevistas en profundidad. La observación etnográfica permitió insertarse en aquellos espacios y lugares de la experiencia de envejecer en diversos contextos de interacción social; principalmente en los hogares de las personas mayores de 90 y de 100 años. Para comprender cómo se imbrican estos procesos de transformación fue importante conocer las trayectorias y narrativas biográficas de las personas longevas. La introspección biográfica en el trabajo etnográfico llevó a comprender y dar un contexto espacio-temporal a los diferentes niveles y relaciones personales, familiares, sociales e institucionales de los sujetos longevos.

El trabajo de campo se realizó en la Región Metropolitana y la Región de Los Lagos, abarcando territorios geográficos y socioculturales muy diversos; por lo que se investigó en múltiples escenarios y escalas de configuración etnográfica. Sin embargo, las distancias geográficas entre los lugares particulares y los entramados culturales de cada uno, no fueron problematizados en dicotomías, contraposiciones o ejercicios comparativos; más bien, fueron pensados en las interconexiones entre los distintos lugares-escalas. En el marco de una etnografía multisituada (Marcus 2001), la visita etnográfica fue la estrategia privilegiada. Las personas fueron visitadas más de una vez; se las acompañaba en sus actividades cotidianas, se pasaba la tarde con ellas y se conversaba sobre sus vidas. La primera y segunda visita fueron, principalmente, para construir relaciones de confianza, conocerse, conversar informalmente y tomar mate, té o café. Recién en la tercera visita, se realizaba el registro con grabadora de 
audio, por lo que el diario de campo fue una herramienta de registro constante. La visita se realizaba en dupla y, muchas veces, el relato también era de a dos. Por lo mismo, la estrategia de escritura incluyó relatos polifónicos, donde se hicieran visibles las voces de las personas presentes en estos encuentros. Estas decisiones metodológicas permitieron una articulación etnográfica (Guber 2013), vale decir, articular la experiencia de campo, la información empírica producida y los referentes teórico-conceptuales de la investigación.

Una primera estrategia de campo fue la construcción de vínculos con las familias y los grupos domésticos de las personas nonagenarias y centenarias, generando etnografías en contextos familiares. Para tomar contacto con ellas, se utilizó principalmente la técnica de bola de nieve o accediendo a informantes-claves a través de instituciones públicas y organizaciones sociales. Para las entrevistas etnográficas, se construyó y utilizó un guión temático, focalizado en la vida cotidiana y trayectorias de envejecimiento de las personas longevas. Se realizó un muestreo teórico-intencional, a través de la selección de los participantes en base a sus características estructurales, tales como la edad cronológica: más de 90 y más de 100 años; el contexto geográfico-cultural: zonas urbanas de la región Metropolitana y zonas rurales en la región de Los Lagos; y el género: hombres y mujeres. Fueron criterios de exclusión el estar institucionalizado y/o presentar claros signos de deterioro cognitivo, o alguna demencia diagnosticada. Se tomó contacto y se realizaron visitas etnográficas a 39 personas nonagenarias y a 10 personas centenarias entre ambas regiones.

\section{MANIFESTACIONES DE AGENCIA EN PERSONAS NONAGENARIAS Y CENTENARIAS}

Para observar las manifestaciones de agencia en sujetos nonagenarios y centenarios, se identificó un proceso compuesto por: (a) asimetrías (Archer 2003; Navarro 1996); (b) reflexividad (Archer 2003; Navarro 1996; Giddens 1990); y (c) decisiones (Elder y Johnson 2003). La "asimetría" se refiere al reconocimiento, por parte de un agente, de una experiencia de diferencia de poder en las relaciones con otros agentes de su entorno. Este reconocimiento puede expresarse a través de un lenguaje verbal y/o no verbal, con el cual la persona longeva identifica la heterogeneidad de posiciones estructurales que se asumen en la vida cotidiana. Esta acción de identificar la asimetría en las posiciones son el índice de una "reflexividad" en el sujeto, es decir, de la capacidad agencial de un individuo de elaborar una representación de la realidad social en que habita (Navarro 1996). El sujeto longevo reflexiona sobre esa posición y posteriormente, el desenlace es una "decisión", que se traduce en la forma en que el longevo gobierna (o permite gobernar) su propia vida, independiente de sus niveles de autonomía funcional. 
A continuación se presentan cuatro relatos etnográficos, que forman parte de la construcción de casos realizados a partir de la información etnográfica y que reflejan las diversas formas en que la agencia se configura en la vida cotidiana de personas nonagenarias y centenarias. ${ }^{2}$ Los nombres de las personas fueron modificados, para resguardar su anonimato. Las edades indicadas en la presentación de los casos corresponden a la edad registrada en la primera visita etnográfica. Se utiliza el símbolo de la cruz entre paréntesis para consignar el fallecimiento de estas personas.

\section{Mariluz, 94 años: "Ahora espero a mi vecina pa' [para] ducharme porque me da miedo que se me vaya a torcer la pierna en el baño"}

Mariluz nació en el año 1922 en Santiago de Chile. ${ }^{3}$ Se considera de una familia longeva ya que su madre vivió hasta los 95 años. Hasta cerca de los 50 años vivió con su madre, hasta casarse con un hombre, viudo y con hijos. Producto de su tardío matrimonio, Mariluz no tuvo hijos biológicos; sin embargo, adoptó el papel de madre, y las personas que ella llama hijos, nietos y bisnietos devienen de esta alianza matrimonial. Viuda hace 25 años, continúa habitando la misma casa, en un municipio de clase media de la Región Metropolitana. La imagen de la viudez, a pesar de haberse consolidado hace varios años, la hace normalizar su actual situación. Producto de reiteradas caídas, y de la fragilidad de sus piernas - al doblarse de manera inesperada -, ha perdido la confianza para salir a lugares públicos de forma independiente, teniendo que recurrir a familiares o vecinas que la acompañen. Este temor lo ha trasladado a la intimidad de su hogar, pidiendo compañía en los momentos en que ingresa a la ducha, por miedo de tropezar y caerse en ese instante. Prefiere contactar a terceras personas cuando necesita algún tipo de ayuda, en vez de contratar servicios formales para su cuidado.

Mariluz logra generar una red de apoyo que va más allá de los lazos familiares. Estas redes afectivas y sociales le permiten cubrir sus necesidades cotidianas. Como ella misma relata: "me atreví la semana pasada, que me acompañó la señora que vive frente a mi casa, a ir yo al supermercado, pero ya este mes no voy a ir porque estoy mal. Porque como que no me dan ganas de ir o hacer las cosas que hacía antes". Mariluz enfrenta quiebres en su rutina debido a sus malestares corporales y emocionales. Las vecinas, mujeres claves en estos procesos de recomposición de situaciones, entregan a la nonagenaria la posibilidad de realizar de otras maneras sus actividades cotidianas. Tanto es el nivel de confianza y de apoyo que obtiene Mariluz de sus vecinas, que estas cuentan con una copia de llaves de su hogar.

2 En las construcciones de caso, coexisten más de una manifestación de agencia; sin embargo, acorde al caso se señala aquella más representativa para los efectos de este trabajo.

3 La primera visita etnográfica a Mariluz ha sido realizada en junio del 2016. 
Su rutina diaria comienza cerca de las 9:00-9:30 horas de la mañana, aunque en invierno y/o cuando está enferma se permite quedarse hasta un poco más tarde recostada. Cuenta:

"Barro algo para que se vea limpio, sacudo algo si hay que sacudir, pero se me va la mañana, así que vengo a tomar desayuno como a las 1 1:00 porque no me gusta tomar desayuno sin haber hecho la cama y como me levanto a las 9:00, en ducharme, qué sé yo. Ahora espero a mi vecina pa' [para] ducharme porque me da miedo que se me vaya a torcer la pierna en el baño. Así que esa es mi rutina" [Mariluz].

Mariluz genera una reflexión frente a su situación de fragilidad y temor a caerse, y desarrolla una acción movilizando a otros para re-estabilizar su rutina. Junto con el desayuno toma sus medicamentos para la presión, el colesterol y la artrosis. Luego prepara almuerzo para dos o tres días y en la tarde programa alguna actividad, como asistir al centro de salud primario, ir a diversas terapias alternativas, agendar hora con la peluquera (quien va a la casa) o recibir alguna visita. Cerca de las 18:00 horas prepara el té y vuelve a tomar sus medicamentos. Al acercarse el fin de semana, prepara repostería para entregar a sus bisnietos, aprovechando que su familia la visita o la pasan a buscar para paseos.

Para el cobro de su pensión y la realización de algún trámite, ella debe ir personalmente, a pesar de sus deseos de delegar esta responsabilidad en su nieta. Mariluz aún no ha podido generar el poder notarial que le permita desligarse de esta responsabilidad institucional.

Hasta el año 2015, Mariluz participaba activamente de talleres para personas mayores:

"Iba a gimnasia, iba a reuniones, iba a bachillerato, iba... Pero ahora no. No. Ahora no, más ahora que se me tambalean las piernas. No ve que me puse ¿cómo le diré? Media ermitaña creo yo [...] Por ejemplo, ahora aquí hay mucha actividad en un club grandote que está por aquí cerquita, me invitan, ahora me tenían pa' mañana para ir a un bachillerato, pero no me nace" [Mariluz].

Aunque expresa que ya no le motiva participar de las actividades, de igual forma es una mujer muy sociable, dispuesta siempre a conversar y asistir a diversas instancias grupales. Por recomendación de la kinesióloga, cursa un taller sobre "el arte de envejecer" en donde realiza ejercicios físicos y de estimulación cognitiva. En estos talleres, Mariluz socializa y tiene contacto con otras personas mayores. Como tiene bien claro, ella es siempre la más vieja.

El relato que Mariluz genera entorno a su cotidianeidad da cuenta de una manifestación de agencia bajo la forma de "movilizar". El miedo es un nuevo 
componente en su rutina diaria. Para enfrentar este elemento disruptivo, Mariluz activa roles y relaciones, tanto familiares como sociales, en función del reconocimiento de sus limitaciones. Reflexiona frente a estas limitaciones y temores, sentidos y vividos tanto en el espacio doméstico como en el público. Ya ni su casa ni la calle son lugares de seguridad y confianza para ella. Frente a esto, genera estrategias orientadas a recomponer circunstancias de su vida cotidiana, movilizando a otros/as: obtener ayuda para ducharse o para ir a comprar al supermercado. Mariluz vive en un barrio donde ya es una vecina conocida, lo cual es una herramienta para visibilizarse socialmente. Sus redes barriales la observan como una persona que necesita de pequeñas ayudas, más aún al vivir ella sola. Que las vecinas tengan una copia de las llaves de la casa de la nonagenaria, es una acción ilustrativa de la movilización de esta red de apoyo. "Ahora espero a mi vecina pa' [para] ducharme, porque me da miedo" enseña estrategias elaboradas a partir del miedo y del reconocimiento de la existencia de un vínculo social de cuidado. Más que un sentimiento que anule facultades de racionalización o toma de decisiones, el miedo es el motor que impulsa una reflexividad en Mariluz. En las palabras de Mariluz, están descritas sus necesidades y lo que hace para satisfacerlas. Mostrando o expresando lo que necesita, logra movilizar a otros/as para establecer una estabilidad en su día a día.

\section{Mercedes, 95 años (†): "Si tuviera 80 años, estaría trabajando, iría a todos lados sola y mi vida sería muy diferente"}

Mercedes vive en una casa de madera, con tres habitaciones, en una población popular del área urbana de Puerto Montt, Región de Los Lagos. ${ }^{4}$ Nació en 1920, es viuda hace 15 años, vive con un hijo separado de 66 años y otros familiares. Normalmente, viste chalecos de colores y un delantal de cocina sobre la ropa. Pasa la mayor parte del día en la cocina, que también funciona como comedor. Casi al centro del lugar, junto a una ventana, hay una cocina a leña. Arriba de ella, en un rincón, ropa tendida para secar. En las primeras conversaciones guiadas por la pauta de entrevista, en las pausas largas, Mercedes introducía temas de su interés, o contaba cómo era su vida. Ella se casó muy joven, a los 20 años, con su primer marido. Se arrepiente hasta el día de hoy, pues dice que no lo pasó bien: "la suegra era una mujer difícil y a mi marido a veces le gustaba tomar [beber]".

Reiteradamente señala que ella hace todas sus cosas, aludiendo a sus responsabilidades domésticas, aunque "a veces las nietas o bisnietas me ayudan a limpiar la casa... pero no me gusta que otras cocinen”. Cocinar es algo que le gusta hacer sola. A pesar de que se sabe muy activa y autónoma, reconoce que ha habido cambios: "ya no trabajo. Tejía medias para vender, pero ahora ya no 
lo puedo hacer porque me duele el cerebro [sic] y luego el dolor se me va a los ojos". Si bien ya no teje ni vende medias de lana, genera otros ingresos económicos, como hacer mermelada casera y venderla a conocidos. Frente al dolor ocasional en su visión, ha dejado de ir al centro de la ciudad sola. Se desorienta y le da miedo caerse. En general, cuando sale a la calle, lo hace con alguna nieta o bisnieta. Va acompañada a cobrar la pensión, al centro de atención primario de salud y a la feria a comprar frutas, verduras, carnes y mariscos. En términos cronológicos marca una diferencia: "si tuviera 80 años, estaría trabajando, iría a todos lados sola y mi vida sería muy diferente".

Todos los días prepara almuerzo, para ella y los familiares que viven en la misma casa. Le gusta escuchar "los avisos" de la Radio Reloncaví, a las 12:00 del día, para saber si algún conocido ha muerto. Asiste regularmente al club de adulto mayor. Para ir a estas reuniones, dedica tiempo para arreglarse: se cambia los zapatos y ordena su peinado. Antes de salir de su casa, suele revisar si ha dejado la radio u otros objetos de valor encima de la mesa de comedor. Tiene problemas con su bisnieto, quien le saca sus objetos personales. También deja la puerta de su habitación cerrada con un candado.

A través del diálogo entre el discurso de Mercedes sobre su no hacer, y la observación etnográfica donde se observa el control sobre elementos de su cotidianidad, se da cuenta de una manifestación de agencia bajo la forma de hacer/no hacer. El quiebre que genera una asimetría en la posición estructural de la longeva es su edad cronológica: "si tuviera 80 años [...] mi vida sería muy diferente". La percepción de Mercedes sobre su vida actual pareciera acoplarse con la metáfora del hoyo negro de la cuarta edad; las actividades cotidianas se ven mermadas por malestares corporales, impactando su auto-percepción en cuanto persona independiente y autónoma. Esta situación ciertamente genera una incomodidad en Mercedes, hay algo que ya no es como antes. Para enfrentar estos sucesos, la nonagenaria reflexiona y genera conjuntos de reglas - o cursos de acción - para llevar a cabo su vida cotidiana. El "hacer" sería una articulación de estrategias orientadas al control de la situación que experimenta Mercedes; "no hacer" refiere a una articulación de estrategias orientadas a reconocer la asimetría en la situación que experimenta la persona. Dentro de esta lógica, el "no hacer" no significa "no ser". ¿Qué ha cambiado para Mercedes? Algo dice desde la reflexividad de su posición estructural: "mi vida sería muy diferente". Lo que es diferente es el dejar de hacer como en su vejez de 80 años. Y lo que es diferente es que la experiencia de su longevidad difiere con otros momentos de su vida, lo que en ningún caso representa una pérdida de agencia, pues ella reflexiona sobre eso y genera estrategias para recomponer su cotidianeidad.

Mercedes, a través de su rutina cotidiana, mantiene labores domésticas de las que tiene absoluto control. La cocina aparece como espacio de dominio, encargándose principalmente del almuerzo familiar. La toma de decisiones 
respecto a su vida, es de ella. Se identifica en su discurso la auto-percepción de que ella "ya no" trabaja, al existir un cese en sus actividades productivas-económicas tradicionales en su curso de vida, como era la producción de tejidos. Sin embargo, continúa elaborando productos para la venta, como son las mermeladas, lo cual pareciera tener otro valor simbólico en cuanto actividad laboral e ingreso económico. Otro punto importante es el contexto habitacional y familiar en que vive. Al tener problemas con sus bisnietos jóvenes, diseña estrategias para resguardar sus objetos personales, ante posibles hurtos.

\section{Bernarda, 98 años (†): "No me gusta salir, eso del bastón, que la vean del brazo, que 'ay la abuelita'"}

Bernarda nació en 1917, en Tierra del Fuego, Región de Magallanes. Proviene de una familia con ascendencia Croata. ${ }^{5} \mathrm{Al}$ migrar a Santiago siguió recordando sus orígenes y ha participado toda su vida - aunque de manera esporádica en los últimos años - en el Club Croata. La familia, para Bernarda, es un factor relevante: "vivo con mi hijo, él vive al lado, así que estamos juntos, pero no revueltos. Así que de todas maneras, estoy bien acompañada [...] En ese sentido, Dios me ha premiado harto. Porque estoy súper acompañada a esta altura de la vida".

Habita sola en un municipio de clase media-alta de la Región Metropolitana. La casa por lo general no se encuentra vacía. Durante el día, tres veces a la semana, Bernarda pasa el tiempo con la "nana" ${ }^{6}$ y constantemente entra y sale gente de la casa. Consciente de su vida y del apoyo familiar que ha tenido, suele referirse con cariño hacia los integrantes de su familia. A pesar de este ánimo y compañía que Bernarda expresa sentir, se nota en su mirada, en sus palabras y en su andar una pesadez, una añoranza y un cansancio: "uno se siente un poco cansada. A pesar de que una está rodeada de todos sus hijos, toda su familia. Una, de repente, igual siente como nostalgia. Como pena porque ahora se fueron todos mis hermanos menores, entonces uno vive muchos recuerdos, pero, en fin".

Los días en que la "nana" no va a trabajar, Bernarda prepara su desayuno y hace lo que puede en la casa. Intenta mantenerse "lo más independiente posible", pues de acuerdo con lo que ella siente y expresa: "yo no molesto a nadie, yo hago mis cosas, lo que tengo que hacer, dentro de la que puedo. Hago mis cosas, me levanto, me sirvo mi desayuno, en la tarde me sirvo mi té. El almuerzo no, porque siempre están las niñas que sirven el almuerzo".

$\mathrm{Al}$ interior de su hogar, Bernarda se mueve y se desplaza con bastante facilidad, conoce el lugar y sabe dónde encontrar puntos de apoyo. Bernarda reconoce que desde que le vino un mareo hace unos cuatro años y tuvo una

5 La primera visita etnográfica a Bernarda ha sido realizada en mayo del 2016.

6 "Nana": chilenismo para nombrar a las trabajadoras domésticas. 
fractura al fémur, ya no sale sola, porque "ya no puedo y tampoco me dejan". Además, comenta que "como están las calles, cuesta mucho". En la calle no sabe los obstáculos que se podrá encontrar, de dónde obtener apoyo si lo necesita o qué hacer en caso de que le venga un mareo que la haga caer al suelo; por tanto, decide abandonar la idea de salir sola. Ha reducido sus actividades sociales e incluso familiares, dejando de visitar a su hermana menor. Para recomponer su relación, Bernarda utiliza el teléfono como medio para mantener vínculos.

Si bien es una mujer que no se queja por su salud ni habla de sus dolencias, afirma ser operada de cataratas en un solo ojo, ya que no quiso operarse el otro. Tiene visión en un ojo, de igual forma que escucha solo por un oído. Esta omisión de las enfermedades o dolencias se debe a su forma de entender la vejez, como una etapa en la que ella, personalmente, prefiere conformarse y no quejarse, como estrategia para no preocupar a sus cercanos: "no hay que molestar a los jóvenes, uno no les puede cortar la vida".

La cotidianidad de Bernarda es bastante "fome", ${ }^{7}$ como ella la define. Se levanta, viste, y arregla con tranquilidad. Se toma el tiempo para vestirse bien, siempre combinada y maquillada. Luego toma desayuno, camina un poco por la terraza y después se sienta, lee o ve televisión. A veces teje, aunque últimamente esta actividad no le ha llamado tanto la atención. Llegada la hora de almuerzo, la visitan su nuera y su nieta. Ya para la tarde se sienta frente a la televisión y toma una siesta o ve las teleseries. En la noche, cerca de las 21:30 o las 22:00 horas, Bernarda se acuesta y lee hasta dormirse.

La nonagenaria confía en la "nana" para acompañarla en los paseos por el barrio. Caminan tomadas del brazo, sobreponiéndose con esto a la imagen que le desagrada generar al salir, la de la "abuelita desvalida", pues como ella misma cuenta: "no me gusta salir, eso del bastón, que la vean del brazo, que 'ay la abuelita'".

¿De qué habla Bernarda? ¿Qué hay en sus omisiones cotidianas? La longeva manifiesta su agencia al "resistir la posición" que ocupa como persona muy mayor en su entorno familiar y social. Como se ha enunciado, la estructura normativa y los imaginarios de la cuarta edad aluden a la fragilidad, dependencia, cercanía a la muerte y entre otros (Gilleard y Higgs 2011). Bernarda genera una resistencia a los imaginarios que reviste esta categoría etaria. Mientras que la cuarta edad no representa una estructura per se, es un constructo social que existe en la intersección de estructuras poderosas, prácticas organizacionales y constreñimientos experimentados por las personas mayores (Grenier y Phillipson 2013). Bernarda identifica la asimetría, reflexiona y su férrea oposición tiene sentido en cuanto logra vislumbrarse desde "resistir su posición" en esta estructura de relaciones y de normalización social. 
La casi centenaria reiteradamente menciona lo "fome" que es su rutina. También su resistencia está presente en esta expresión de aburrimiento. ¿Qué es lo aburrido? Pareciera que es el deber ser de la vejez avanzada lo que no atrae la atención de Bernarda. Mantener vínculos familiares cercanos es un aspecto con el que contrarresta su cotidianeidad. No se trata de catalogar si Bernarda vive su longevidad de manera feliz o infeliz, más bien, es la reflexividad que la lleva a no contarles a sus familiares sus pesares o molestias; como ella dice, "no hay que molestar a los jóvenes, uno no les puede cortar la vida". Si bien sabe que no puede hacer desaparecer la vejez avanzada de sí misma, sí puede omitir aquello que de ella pareciera molestar socialmente. Esto, entendido como resistencia, también se extrapola al espacio público, al no querer salir a la calle junto a la "nana" del brazo, para no ser catalogada dentro del imaginario social de "la abuelita desvalida". Las condiciones arquitectónicas y urbanas acompañan la pérdida gradual de su autonomía, situando la calle como lugar non-grato para una persona que requiere de asistencia al desplazarse, como ella.

Juvenal, 92 años ( $\dagger$ ): “No hago na' [nada], estar en la casa sentao [sentado]"” En Chaica, localidad rural del municipio de Puerto Montt, Región de Los Lagos, transcurre una vida calma y amena para Juvenal, nacido en $1923 .{ }^{8}$ Es un hombre soltero, ya que decidió no emparejarse ni tener hijos. Vive con su sobrina nieta y la familia de ella. Para llegar a su hogar, hay que cruzar varios cercos y subir por una pequeña ladera. La entrada a la casa está custodiada por un perrito y una chancha de tres o cuatro meses, tratada como una mascota más. En el primer encuentro, Juvenal esperaba nuestra visita. A un costado de la cocina a leña estaba Juvenal sentado en un sillón, donde habitualmente pasa el día. Lo primero que llama la atención en él es que le falta su ojo derecho y, al parecer, poco ve por el izquierdo. Usa gafas para ver mejor. Su caminar es lento, pero seguro. Viste un pantalón de tela y un chaleco, además de llevar una boina. Su piel es morena y su pelo cano se matiza con el oscuro. Tiene dificultades de modulación, auditivas y visuales, por ello es difícil aplicar una pauta de entrevista o grabar la conversación en audio digital. El diario de campo resulta clave en este encuentro.

Desde que él perdiera la vista en uno de sus ojos, hace unos años atrás, por un glaucoma no tratado a tiempo, su sobrina nieta realiza los trámites por su tío. Juvenal le dio un poder notarial para que ella cobre su pensión mensualmente. No sale solo porque su familia teme que le ocurra algo. Él participaba de un club del adulto mayor de la localidad y, en el último tiempo, la sobrina nieta lo acompañaba. Sin embargo, dejó de asistir a las reuniones y los paseos cuando su sobrina nieta comenzó a trabajar en una industria de salmones y 
sus horarios le impedían seguir acompañándolo. Juvenal dice que él ya no hace tantas cosas. Dejó de cuidar los animales y ya no puede trabajar el campo. Todavía corta leña y la lleva para la cocina. Eso le gusta hacer. Sin embargo, la sobrina nieta dice que cada vez que lo pilla cortando o llevando leña, ella se lo impide. No lo deja, pues se puede caer o hacer daño. A Juvenal le gusta hablar y contar eventos de su vida. De más joven, trabajó mucho en la construcción y haciendo carreteras. Él es un nonagenario muy lúcido y se recuerda muy bien de situaciones tanto pasadas como presentes.

Como vive al pie de un cerro, lo que más lo aqueja es el frío, sobre todo en invierno. Cuando le preguntamos por su actividad cotidiana, responde: "no hago na' [nada], estar en la casa, sentao' [sentado]". Dice que tampoco puede caminar mucho, ya que se le acelera el corazón. Aunque esté "sentado" en la casa, todos los días se levanta entre 8:30-9:00 horas de la mañana, y en invierno continúa con la misma rutina. Va poco a Puerto Montt, y sobre ir a la ciudad dice: "no me animo a ir al pueblo, me puedo perder". Chaica es "el lugar que conoce"; y al ser el lugar donde Juvenal nació, vivió su juventud, adultez y vejez, es un lugar de protección y seguridad para él también.

Su mundo es el espacio doméstico, que no sólo corresponde al interior mismo del hogar, extendiéndose la vida doméstica hacia el exterior: el trabajo con la tierra, crianza de animales y relacionamiento directo con la naturaleza. Aunque Juvenal ya no realice acciones directas - físicas, principalmente - en este espacio, él está presente, atento y alerta a todo lo que ocurre; cuándo es tiempo de sembrar, cultivar; qué animales hay en la casa y cómo hay que cuidarlos; qué pasa con el mar y las mareas.

En él hay una manifestación de agencia vinculada a la reflexividad del leitmotiv biográfico de la persona; lo que ha estado presente en la trayectoria vital-laboral, persiste y continúa en la longevidad. Por lo mismo, Juvenal es un hombre que "normaliza su posición", al estabilizarse dentro de la estructura normativa de la vejez avanzada. A priori, la estrategia que conlleva es que la persona longeva acepta su posición en la estructura que se le impone. Al respecto, se identifican ciertos alcances. Primero, la normalización depende de la posición estructural del longevo: ¿quién es?, ¿cómo se compone su familia?, ¿cuáles son sus condiciones actuales de vida? Es decir, es necesario indagar en su contexto cotidiano y biográfico. Segundo, se presenta una reflexividad con la representación de las normas de la edad (Neugarten 1999), donde la persona longeva se acopla a su edad cronológica como estructura. La edad en la vejez avanzada, no desaparece, más bien, localiza a la persona en un determinado lugar de la estructura social.

Por su situación de hombre soltero sin hijos/as, Juvenal llega a vivir a la casa de su sobrina nieta. Al tener problemas de movilidad, audición y visión, Juvenal se transforma en un sujeto de cuidados, y él se sabe así, cuidado. Su sobrina nieta ya no lo deja hacer algunas actividades que tanto le gustaban a 
él hacer, como cortar leña. Juvenal debe personificar y encarnar una actitud pasiva, de "no hacer nada". Al normalizar la estructura de cuidados impuesta, también genera acciones de acatamiento. Juvenal reflexiona sobre su propia situación (de asimetría), frente a lo cual evalúa y decide evitar el conflicto con la persona de la que depende, o de quienes recibe ayuda cotidianamente. Él normaliza esa posición devenida de la estructura normativa de la vejez avanzada. Sin embargo, su sobrina nieta dice que a veces "lo pilla" cortando leña. La reflexión gira frente al uso discursivo que Juvenal otorga a estas acciones, donde al contrario del caso de Bernarda, la omisión permite esconder aquel hacer que rompe con su imagen de longevo-cuidado.

\section{PALABRAS DE CIERRE: LA AGENCIA EN LA LONGEVIDAD A TRAVÉS DE LA ETNOGRAFÍA}

En este artículo se han abordado manifestaciones y particularidades de la agencia en la longevidad en contextos rurales y urbanos. El trabajo de campo y análisis de sus resultados abren focos de conocimiento e investigación en torno a la controversia de la agencia en la cuarta edad. A través de relatos de casos etnográficos, se plantearon lineamientos de cómo observar y comprender manifestaciones de agencia de personas nonagenarias y centenarias. Tras tres años de trabajo de campo, es posible destacar la etnografía como método que permite acercarse a la complejidad de la agencia en la longevidad, integrando elementos socioculturales y relaciones establecidas entre las personas mayores y sus contextos (personales, familiares, sociales e institucionales).

En términos metodológicos, el establecimiento de vínculos y las reiteradas visitas etnográficas permitieron ahondar en situaciones que podrían omitirse por medio de una entrevista semi-estructurada, por ejemplo. Por ello, la agencia ha sido comprendida mediante un trabajo etnográfico en el que no sólo se profundizó en la trayectoria biográfica y experiencia de la longevidad de la persona entrevistada, ya que este eje llevaría a centrarse en el individuo longevo como sujeto aislado. Más bien, observando las interrelaciones de la persona longeva con su entorno familiar, social e institucional, se accedió a las posiciones estructurales desde donde las personas nonagenarias y centenarias enuncian sus discursos y manifiestan su agencia. La etnografía intencionada en los contextos en que las personas hablan de sí a través de otros marcos de interpretación, como en recorridos por los espacios domésticos, clubes de adulto mayor, referirse a objetos de asistencia, entre otros, da pie a lecturas a través de recorridos sensoriales y espaciales: "estar ahí", en los contextos de interacción y donde ellas viven.

La dimensión relacional está presente en la construcción que hace la persona longeva de sí misma, distinta a las construcciones narrativas que hacen otras personas - familiares, vecinos o profesionales de la salud - sobre la vida 
del/de la longevo/a. En la multiplicidad de discursos, no se debe privilegiar uno u otro; más bien, tomar en cuenta todos los discursos que giran alrededor del/de la longevo/a. El registro de campo permitió dar cuenta de que cuando los/as longevos/as hablan de lo que ellos/as quieren hablar, se abre una perspectiva para comprender cómo la persona se posiciona dentro de un contexto y narrativa particular, tal como queda expresado en el siguiente extracto etnográfico:

"Al retomar nuestro concepto eje de investigación, la agencia, reflexioné que ésta está presente en la construcción que hace la persona longeva de sí misma desde un determinado discurso. Discurso muy distinto al discurso con que la familia o los más cercanos construyen al nonagenario o centenario. En esos contrapuntos hay resistencia (Bernarda). Lo importante era reflexionar sobre de qué me hablaban esos discursos aparentemente contradictorios. Entendí que me hablaban de manifestaciones de agencia de hombres y mujeres longevas; de la resistencia para no transformar en norma su posición envejecida en la vida y ante su entorno directo, principalmente, con quienes viven o de quienes 'dependen' diariamente. Las personas nonagenarias y centenarias sabían cuáles eran sus limitaciones. Varios decían 'ahora me canso más'; 'ya no es lo mismo que antes'; 'pasan los años'; 'no abuso del tiempo'. Pero también sabían cuáles eran las limitaciones que construyen otros desde fuera: 'me tratan más como niño'; 'me cortaron las alas'; 'no me dejan hacer nada'. Es en ese cruce donde la persona longeva, por un lado, negocia y, por otro, utiliza estrategias (Juvenal). En estas últimas, emerge la agencia, manifestándose de diversas formas" [cuaderno de campo, Región de Los Lagos, 11 noviembre del 2015].

Otro aspecto a considerar, emergente luego de las primeras visitas etnográficas, y en la escritura y análisis de información, se relaciona a cómo se construyen los casos etnográficos y las manifestaciones de agencia presentes en estos. Los prejuicios a partir de los aspectos muy envejecidos de las personas longevas, donde a veces se presentan dificultades comunicativas y/o de movilidad, pudieron haber generado distanciamiento respecto a los "casos etnográficos". Estos prejuicios provienen de un sesgo y visión "adultocéntrica", propia de la conceptualización de la agencia en la cuarta edad como "hoyo negro" (Gilleard y Higgs 2010). En Chile, existe evidencia cuantitativa respecto de estos prejuicios que muestra que la mayoría (68\%) de la población considera que, en el segmento más envejecido, las personas mayores son incapaces de autovalerse (Arnold et al. 2018: 126). Aquí, la alerta etnográfica se centra en el tiempo requerido para trabajar con personas longevas, desplazando nociones a priori de lo que es la agencia en sí. No habría una única perspectiva sobre la agencia, por ello no hay que cerrar el concepto en sí, y 
no buscar la agencia que el etnógrafo quiere escuchar y encontrar. Hay que situar a la persona longeva en un universo temporal, espacial, relacional y corporal distinto.

De acuerdo a los casos etnográficos analizados, la agencia en la longevidad se configuraría de acuerdo a las características contextuales y a la posición estructural de las personas nonagenarias y centenarias. Es así en el caso de Mariluz: ella moviliza a otros desde su posición de mujer urbana, viuda sin hijos, con fuertes vínculos vecinales de una comuna de clase media de la Región Metropolitana. Esta proximidad social se da en un contexto urbano de un barrio tradicional, cuyas características están presentes en las acciones que Mariluz genera respecto a su manifestación de agencia de tipo "movilizar". La peluquera va a la casa de Mariluz cuando ella lo necesita, o la nonagenaria es invitada a diversas actividades recreativas o de participación social en su territorio. Las redes, vínculos y afectos son con otras mujeres, lo que da cuenta de cómo la categoría de género emerge en la posición estructural de Mariluz. $\mathrm{Al}$ respecto, hay diferencias para hombres y mujeres mayores en la articulación de redes comunitarias. La literatura señala que mientras para los hombres el apoyo social proviene de la familia, las mujeres mayores generan redes no familiares y de confianza con otras mujeres, en comparación con hombres de la misma edad (Wenger 1996, en Montes de Oca 2003).

La vida cotidiana de Mercedes es diferente. Su posición estructural nos habla de una mujer cuya trayectoria biográfica se cruza con una precariedad social y económica. No profesional, dueña de casa, viuda y artesana. Vive en una población urbana de la ciudad de Puerto Montt, de alta vulneración social. Es la posición de una mujer pobre que ha tenido que trabajar durante toda su vida, además de encargarse del trabajo doméstico y cuidado de familiares, labores que continúa realizando. Aquí, la interseccionalidad de la categoría género, habla. Es así como su manifestación de agencia de "hacer/no hacer" se condice con la discontinuidad de su hacer actual, donde hace menos que años atrás.

Las características de Bernarda en cuanto mujer urbana, viuda y ama de casa, la posiciona en una relación con su familia de vínculos cercanos, pero que establece límites para su independencia, como es decidir vivir sola, reducir sus actividades sociales y dejar de salir sin compañía. A través de estas decisiones, ella manifiesta su agencia "resistiéndose" a su situación de mujer longeva y resistiéndose también a los elementos simbólicos de la vejez avanzada, como la necesidad de usar un bastón o de tener que salir a la calle en compañía de un otro.

Juvenal normaliza su posición en cuanto hombre soltero y sin hijos, que por su edad avanzada construye una relación de dependencia con su sobrina nieta, en un contexto rural. La ruralidad como marcador territorial ha estado presente a lo largo de la vida de Juvenal y en su experiencia de envejecimiento 
avanzado como un continuum. De tal forma que su vida cotidiana se organiza con los tiempos y rutinas vinculados a la ruralidad. Al igual que Bernarda, él reconoce cambios y cosas que ha dejado de hacer. El recorrido de su trayectoria como hombre longevo muestra de qué forma va "normalizando" su posición de vejez: dejando de ir al club, delegando en otros varias de sus acciones y decisiones, como en el caso de la sobrina nieta que le cobra la pensión. Se acopla a esta nueva posición estructural de vivir en casa de su familiar y de depender de sus cuidados. Esta realidad se condice con los patrones de cohabitación y convivencia intergeneracional en Chile, al haber un mayor porcentaje en zonas rurales $(44,8 \%)$ de hogares que tienen algún integrante de 60 años y más, frente a las zonas urbanas (39,4\%) (MDS 2017).

Por último, los resultados muestran que no necesariamente hay una relación entre el deterioro de las capacidades físicas y funcionales y la reducción/ pérdida de agencia, sino, más bien, que es el contexto de las relaciones sociales y las diversas posiciones estructurales que adquieren los más envejecidos (Osorio-Parraguez y Seguel 2014) lo que inhibe o constriñe las manifestaciones de agencia en la cuarta edad. De esta forma, a través de la etnografía se buscó describir cómo la reflexividad presente en las personas nonagenarias y centenarias se despliega como manifestación de agencia a través de decisiones, estrategias, resistencias, elecciones, proyecciones y movilizaciones de redes sociales en su vida cotidiana, con sus contextos sociales, personales, familiares e institucionales cercanos. 


\section{BIBLIOGRAFÍA}

ARCHER, Margaret S., 1996, Culture and Agency: The Place of Culture in Social Theory. Cambridge, Cambridge University Press.

ARCHER, Margaret S., 2003, "Introduction: how does structure influence agency?", en M. S. Archer, Structure, Agency and the Internal Conversation. Cambridge, Cambridge University Press, 1-16.

ARNOLD, Marcelo, et al., 2018, Quinta Encuesta Nacional de Inclusión y Exclusión Social de las Personas Mayores en Chile: Opiniones de la Población Chilena Respecto al Envejecimiento. Santiago de Chile, SENAMA.

ELDER, Glen H., y Monica K. JOHNSON, 2003, “The life course and aging: challenges, lessons, and new direction”, en R.A. Settersten (org.), Invitation to the Life Course: Towards New Understandings of Later Life. Amityville, NY, Baywood Publishing Company, 49-81.

FLETCHER, Amber J., 2017, “Applying critical realism in qualitative research: methodology meets method", International Journal of Social Research Methodology, 20 (2): 181-194, DOI: $10.1080 / 13645579.2016 .1144401$.

GEERTZ, Clifford, 2005, La Interpretación de las Culturas. Barcelona, Gedisa.

GIDDENS, Anthony, 1990, Consequences of Modernity. Stanford, Stanford University Press.

GILLEARD, Chris, y Paul HIGGS, 2010, "Aging without agency: theorizing the fourth age", Aging \& Mental Health, 14 (2): 121-128, DOI: 10.1080/13607860903228762.

GILlEARD, Chris, y Paul HIGGS, 2011 , "Ageing abjection and embodiment in the fourth age”, Journal of Aging Studies, 25 (2): 135-142, DOI: 10.1016/j.jaging.2010.08.018.

GRENIER, Amanda, y Cristopher PHILLIPSON, 2013, "Rethinking agency in late life: structural and interpretive approaches", en Jars Baars et al. (orgs.), Ageing, Meaning and Social Structure: Connecting Critical and Humanistic Herontology. Bristol, Policy Press, 55-80.

GUBER, Rosana, 2013, La Articulación Etnográfica: Descubrimiento y Trabajo de Campo en la Investigación de Esther Hermitte. Buenos Aires, Editorial Biblos.

INE - INSTITUTO NACIONAL DE ESTADÍSTICAS, 2017, "Primera entrega de resultados definitivos, Censo 2017: cantidad de personas por sexo y edad", disponible en < http:// www.censo2017.cl/wp-content/uploads/2017/12/Cantidad-de-Personas-por-Sexo-y-Eda d.xlsx > (última consultación en octubre del 2019).

KAWAS, Claudia H. , 2008, “The oldest old and the 90+ Study”, Alzheimer's \& Dementia: The Journal of the Alzheimer's Association, 4 (1): S56-S59, DOI: 10.1016/j.jalz.2007.11.007.

LALIVE D'EPINAY, Christian, y Stefano CAVALLI, 2013, Le Quatrième Age ou la dernière étape de la vie. Lausana, Presses polytechniques et universitaires romandes, col. Le savoir suisse.

LALIVE D'EPINAY, Christian, y Dario SPINI, 2007, “Le grand âge: un domaine de recherche récent”, Gérontologie et Société, 123 (4): 31-54, DOI: 10.3917/gs.123.0031.

LASLETT, Peter, 1989, A Fresh Map of Life: The Emergence of the Third Age. Londres, Weidenfeld and Nicolson.

MARCUS, George, 2001, "Etnografía en/del sistema mundo: el surgimiento de la etnografía multilocal”, Alteridades, 11 (22): 11 1-127, disponible en < http://www.redalyc.org/articu lo.oa?id=74702209> (última consulta en octubre del 2019).

MDS - Ministerio de Desarrollo Social, 2017, "Casen 2015: adultos mayores, síntesis de resultados", disponible en < http://observatorio.ministeriodesarrollosocial.gob.cl/cas en-multidimensional/casen/docs/CASEN_2015_Resultados_adultos_mayores.pdf___> (última consulta en octubre del 2019). 
MONTES DE OCA, Verónica, 2003, “Redes comunitarias, género y envejecimiento: el significado de las redes comunitarias en la calidad de vida de hombres y mujeres adultos mayores en la Ciudad de México", Notas de Población, 77: 139-174, disponible en < https://re positorio.cepal.org/handle/1 1362/12753 > (última consulta en octubre del 2019).

NAVARro, Pablo, 1996, "Hacia una teoría de la morfogénesis social”, en A. Pérez-Agote Poveda e I. Sánchez de la Yncera (orgs.), Complejidad y Teoría Social. Madrid, Centro de Investigaciones Sociológicas, 436-465.

NEUGARTEN, Bernice L., 1999, Los Significados de la Edad. Barcelona, Herder.

OSORIO-PARRAGUEZ, Paulina, y Andrés G. SEGUEL, 2014, "Social construction of dependence in elderly men in Chile”, Health, 6 (10): 998-1003, DOI: 10.4236/health.2014.61 0125 .

SOARES, Cristiane Leal Rodrigues, 2014, “Três histórias: velhice feminina, agência e reflexividade em contextos de convivências intergeracionais", Sociedade e Cultura, 17 (1): 17-28, DOI: 10.5216/sec.v17il.36871.

USCB - UNITED STATES CENSUS BUREAU, 2018, "Mid-year population by older five year age groups and sex, custom region: Chile, 2050", en International Data Base, United States Census Bureau, disponible en < https:/www.census.gov/data-tools/demo/idb/region. php? $\mathrm{T}=3 \& \mathrm{RT}=0 \& \mathrm{~A}=$ both $\& \mathrm{Y}=2050 \& \mathrm{C}=\mathrm{CI} \& \mathrm{R}=>$ (última consulta en octubre del 2019). 